Archives

$44 \mid 2009$

Varia

\title{
De la capacité d'inflexion de la cité impériale de
} Besançon

Une décision politique entre souverains lointains :Madrid 1660-1664

\section{Annonciade de Cambolas}

\section{CpenEdition}

Journals

Édition électronique

URL : http://journals.openedition.org/ccrh/3526

DOI : $10.4000 /$ ccrh.3526

ISSN : 1760-7906

Éditeur

Centre de recherches historiques - EHESS

Édition imprimée

Date de publication : 25 janvier 2009

Pagination : 41-57

ISSN : 0990-9141

Référence électronique

Annonciade de Cambolas, « De la capacité d'inflexion de la cité impériale de Besançon », Les Cahiers du Centre de Recherches Historiques [En ligne], 44 | 2009, mis en ligne le 25 novembre 2011, consulté le 19 avril 2019. URL : http://journals.openedition.org/ccrh/3526 ; DOI : 10.4000/ccrh.3526

Ce document a été généré automatiquement le 19 avril 2019

Article L.111-1 du Code de la propriété intellectuelle. 


\title{
De la capacité d'inflexion de la cité impériale de Besançon
}

Une décision politique entre souverains lointains :Madrid 1660-1664

\author{
Annonciade de Cambolas
}

1 La période comprise entre 1668 et 1674 au Comté de Bourgogne souffre d'une sorte d'inexistence historique, enserrée entre deux conquêtes françaises, entre deux appartenances à d'imposants modèles politiques rivaux: la monarchie hispanique à laquelle succédera la monarchie française et ce par deux fois ${ }^{1}$. Pourtant cette périodisation fine, que l'historiographie comtoise survole à grands traits, évoquant énigmatiquement une reprise en main politique par l'Espagne, permet de révéler des ruptures tant déstabilisatrices que fondatrices d'un modèle politique nouveau. Bornée par des conquêtes militaires ${ }^{2}$, ces années offrent l'exemple d'une reconquête juridictionnelle, sous la souveraineté d'une reine régente, Marie-Anne d'Autriche, par la refondation de liens politiques qui cessent alors d'être contractuels. Là est notre objet de thèse, la mise à découvert de la pratique institutionnelle de ces nouveaux liens entre province et monarchie dont la nature sera à préciser. Comment une province, ancien patrimoine héréditaire, après avoir trahi son roi, en prêtant serment à Louis XIV, fut-elle réincorporée après quatre mois d'occupation française? Parlement muselé, États non convoqués, charge de Gouverneur aux pouvoirs certes renforcés, mais offerte à des étrangers au Comté, dépendance accrue en faveur de Madrid et deBruxelles ${ }^{3}$ : ainsi se met en place un nouveau modèle politique et fiscal consacrant la disparition de certains acteurs comtois, doublé d'une réduction brutale du cadre dialogique. La question des révoltes des villes et d'une partie de la noblesse permet d'interroger la viabilité et la légitimité de ce modèle qui prétendait se passer des pouvoirs locaux, tout en renforçant ceux de Bruxelles, afin de mettre en œuvre une politique de conservation du bloc territorial du nord, vestige de l'ancien Cercle de Bourgogne.

Or, lorsque le prince de Condé se présenta aux portes de Besançon le 7 février 1668, exigeant sa reddition, il lui fut répondu avec hauteur que la cité n'appartenait pas au roi d'Espagne. Subterfuge habile ou naïveté politique pour l'obtention d'un gain immédiat? 
Quoi qu'il en soit, la cité signifiait publiquement qu'elle considérait alors comme non valide le Traité de $1664^{4}$ qui portait acte de son intégration au Comté de Bourgogne, fragment de la Monarchie catholique, conclu lui-même après de longues années de négociations avec la couronne castillane. C'est ainsi que lors d'une capitulation séparée d'avec la province, Besançon obtient le déplacement du siège d'autorité qui avait été réduit au seul Gouverneur, dans les murs du Palais Granvelle ${ }^{5}$, ce que Madrid confirme en juin 1668. Là se scelle, sous l'autorité de la force - la conquête française - puis d'une nouvelle légitimité - la reconquête espagnole - l'intégration de la cité impériale libre de Besançon au Comté. Le processus de cette intégration avait été pensé à Munster en $1649^{6}$, accordé au Congrès de Westphalie entre les deux branches des Habsbourg en 1653-1654, puis notifié à la cité en $1659^{7}$. L'autorité de l'Empereur s'effaçant, cette cession devait consacrer la création d'un cadre géographique homogène de plus grand prestige et utilité, transcendant les anciennes frontières juridictionnelles qui avait fait de Besançon une enclave dans cette « isle cernez bouclez de tous costé »

3 L'objet de ces pages, en prologue à mon étude sur la réincorporation de ces territoires au sein de la monarchie hispanique en juin 1668, est d'approcher l'intégration non achevée de la ville la plus peuplée, la plus prospère, dans la province. Éclairer sur le vif cette agrégation non aboutie, malgré un traité conclu entre le roi et la cité, incite à réfléchir sur les modalités de l'exercice de l'autorité de la monarchie catholique, face à deux entités provinciales, elles-mêmes dotées de droits particuliers. En effet, le Comté demeure patrimoine héréditaire, et Besançon, cité impériale libre, est liée néanmoins par un traité de Gardienneté au comte de Bourgogne roi d'Espagne. Le Comté de Bourgogne était rattaché à Bruxelles par un lien plus questionné que reconnu ${ }^{10}$. L'étude du processus d'intégration permettra de mettre en lumière l'articulation de pouvoirs concurrents dans cet ensemble territorial.

4 L'important ici sera la capacité de la cité impériale à infléchir une décision politique, prise entre souverains lointains par la voie de la négociation. En effet, sitôt l'Acte de Cession connu en 1660, Besançon, objet d'un échange sans consultation de son corps politique et donc considéré comme illégal, intervient entre l'empereur et le roi d'Espagne comme un espace politique tiers porteur de droits. Deux de ses quatorze gouverneurs, Hugues Belin et Pierre François Henry sont députés à Bruxelles puis à Madrid en 1661. S'ouvre alors une période de quatre années, pendant laquelle la cité impériale s'emploieà établir avec le roi d'Espagne, son futur souverain, une relation qui n'entame en rien une identité politique définie par le respect de son héritage juridictionnel et de son lien à l'empereur. La négociation avait donc pour fonction première de poser le principe d'une relation de dialogue avec Philippe IV, au sujet de son intégration dans le Comté de Bourgogne. Cette relation enregistre la capacité que la ville entendait avoir acquise par ses droits et par la lettre du roi d'Espagne de 1660 qui l'accueillait dans la gloire de sa puissance, l'assurant du respect de ses privilèges, et l'incorporant pour les « rendre participants des biens et honneurs que nous avons accoutumés d'impartir à ceux qui sont du Corps même de notre Monarchie» ${ }^{11}$.

5 Ainsi, en prenant l'initiative diplomatique, la cité se dote du statut d'interlocuteur légitime. Elle faitconnaître publiquement ses droits anciens, à travers le Grand Mémorial présenté à Madrid en 1661. Mon étude porte, non pas sur le déroulement des ambassades ou les relais que les députés se forgent à la cour de Bruxelles et à Madrid, mais sur la justification écrite, fondement de la représentation politique,que déploie la cité en lien étroit avec ses deux députés. Pour contrer les conséquences redoutées du transfert de 
souveraineté, la cité conditionne son acceptation à un gain politique. Arc-boutée sur son ambition, la cité utilise, parallèlement à la négociation, la cérémonie de prestation de serment comme moyen de coaction. La combinaison de la négociation et du refus du serment de fidélité immobilise l'appareil d'état. Puis la cité impériale parvient à placer le roi en position d'arbitre entre vassaux héréditaires et citoyens nouveaux, comme souverain de chacun, aequer principaliter, comte de Bourgogne, souverain de la monarchie composée ${ }^{12}$.

6 Tout d'abord, il convient de préciser la force de représentation du magistrat qui porte cetteinitiative diplomatique au nom du peuple des citoyens ${ }^{13}$. Celui-ci envoie ses députés afin de faire entendre la spécificité de sa juridiction au service d'un projet politique, fondé par la tradition, mais également nourri par des délibérations municipales poursuivies sur une période de plusieurs années. En effet, pour chaque étape de ce processus diplomatique, à Bruxelles de janvier à mars 1661, puis à Madrid de 1661 à 1663, le magistrat, au cours de ses débats municipaux, fait le choix de poursuivre activement la résistance à toute réduction juridictionnelle, et le combat contre plusieurs entités : Dole, Bruxelles et Madrid ${ }^{14}$. Cette opposition permanente structure en retour la configuration de sa personnalité politique. Chaque 24 juin, jour de la Saint Jean-Baptiste, « au son de la cloche accoutumée ", les citoyens se rassemblent devant l'Hôtel consistorial et réaffirme leur confiance en leurs institutions par une élection par acclamation ${ }^{15}$. Cet acte public renforce l'action et le projet politique bisontin. Les nouveaux gouverneurs, unis aux anciens, les quarante-deux notables et magistrats élus l'année précédente manifestent par là même publiquement, au roi et à la province, la légitimité et la permanence du dessein politique de la cité impériale. Ce faisant, ils font courir un risque aux intérêts de la monarchie en interposant à la décision royale, leur exigence opiniâtre du respect de leurs droits anciens et en réclamant des prétentions nouvelles.

\section{Construction de son particularisme : vesontio quod est maximum oppidum sequanorum ${ }^{16}$}

7 Nous souhaitons, à travers l'examen rapide de ce Grand Mémorial ${ }^{17}$, appréhender comment un pouvoir isolé, brutalement mis en contact avec ce vaste ordre juridique pluraliste organisé autour de son centre lointain qu'est Madrid, défend son particularisme par son arme naturelle qu'est l'écriture juridique. Au long de cette mise en écrit de l'histoire de ses droits, le magistrat va se construire un statut d'interlocuteur en prouvant son appartenance à un universalisme qui admet précisément les particularismes.

8 Le Grand Mémorialdéfend donc devant le roi la conformité de l'échange entre la cité impériale et Frankenthal, avec la préservation de ses privilèges anciens. En particulier celui dont découlent tous les autres, celui d'immédiateté à l'Empereur et donc de non subordination à aucun autre pouvoir ${ }^{18}$, droit reconnu par la formule de «Vesuntio nobis, et sacro romano Imperio Immediate subiecta » repris du Diplôme impérial lors de l'Acte de transfert de 1649. À la lumière de ces pages, nous comprenons que la ville ne se pense pas en terme de minorité mais d'autonomie politique, dans la claire conscience de constituer une entité séparée et différenciée. La juridiction de son magistrat est suprême dans les domaines civil, criminel, militaire et politique ${ }^{19}$, d'ailleurs la cité impériale libre s'adresse au roi en se posant en interlocuteur direct: "Sire, la cité de Besançon représente à Votre Majesté [...] $»^{20}$. Ainsi le magistrat, gardien de la stabilité de son 
système politique, combine dans un même mouvement, son appartenance à une culture commune et son particularisme local. Il inscrit ses libertés dans l'architecture des pouvoirs de la monarchie catholique. Il présuppose donc que la nature et force de la monarchie, tout comme la situation constitutionnelle du Saint Empire, réside dans sa capacité à reconnaître la diversité de ses territoires. Le magistrat informe le futur souverain de son organisation juridictionnelle, mais également des conflits aux frontières avec les anciens ducs et comtes de Bourgogne, afin de tracer un trait d'union entre roi et empereur. Besançon réalise alors un effort considérable d'information et de propagande ${ }^{21}$

9 Lors de cette confrontation, la cité impériale est donc en mesure d'élargir son espace politique en démontrant qu'elle participe d'une même culture par l'emploi de références thématiques et historiques communes. En effet, les Bisontins comme les Espagnols conçoivent leur propre histoire face à celle de Rome ${ }^{22}$. La cité s'appuie sur l'antériorité de sa fondation, comptée en années à partir de celle de la ville éternelle (434 ans avant Rome, 1141 ans avant la naissance du Christ). Faire appel à Rome lui permet d'orienter l'étude du passé en termes mystiques et prétendre ainsi à une forme d'universalité. En un sens, l'Empire de Rome n'est pas mort mais perdure à travers l'esprit des gouverneurs, ce qui en retour les hisse au regard des autres pouvoirs concurrents. Ce recours autorise la cité à dater sa présence dans une culture commune, ius commune. La cité s'affirme comme berceau de la chrétienté dans la province. Le rappel et l'idéalisation de sa genèse joue sur les deux tableaux politiques et religieux en les confondant: c'est sa foi précoce qui par grâce divine lui a permis de conserver ses privilèges et libertés, alors que ses ennemis étaient nombreux et puissants. Le magistrat découvrant son passé au roi en fait force d'autorité car,
toute opération de restitutio [...] va chercher dans le
passé (celui des fondateurs mythiques ou réels)
l'accroissement dont elle sait avoir besoin pour
s'imposer. En faisant d'un avant, un passé ressource
et porteur de précédence, c'est une opération pour
temps de crise ${ }^{23}$.

10 Sa fondation antérieure à Rome, sa juridiction héritée de Rome, son rang de capitale de la Séquanie préservée de toute domination étrangère ${ }^{24}$, lui confèrent des droits inscrits dans la durée qui ne peuvent être niés par cet acte de transfert. Bien au contraire, la remontée dans les temps les plus obscurs, l'inscription de la date de fondation sur une frise chronologique quasi mythique, sont source d'autorité pour éclairer les futurs liens que la cité revendique.

11 Prouver son antériorité lui permet également de penser sa différence et de justifier ses droits particuliers invitant le roi à les reconnaître comme l'avait fait l'Empereur. Se différencier par le rappel de ses libertés lui permet de préciser les contours de son identité politique. Sur plusieurs pages, courent les litanies de son droit municipal ${ }^{25}$. Pour les inscrire dans le creuset de la tradition, sont tissés des liens de citations croisées avec d'autres écrits des lettrés de la province. En particulier ceux de Jean-Jacques Chifflet ${ }^{26}$ dont la fidélité éprouvée au service de la monarchie fut récompensée par l'octroi du titre de Médecin de Chambre de Philippe IV,lors de son ambassade à Bruxelles et Madrid ${ }^{27}$. Le magistrat a recours à l'érudition déployée par Chifflet dans ce grand ouvrage qu'est le Vesontio,paru en 1618. Ce récit historique, porteur de la "haute renommée de la très antique cité métropole des séquoines $\aleph^{28}$, déroule une peinture passionnée de la ville romaine au détriment de la ville médiévale. Cela permet la jointure des deux époques 
glorieuses de la ville, l'époque romaine et l'époque habsbourgeoise ${ }^{29}$. Ce travail d'interprétation de leur histoire récupère une tradition très ancienne, participe à sa construction, et véhicule des stéréotypes qui ravivent la cohésion de la communauté des citoyens de la cité. Le réemploi de ces écrits faisait écho à une ancienne querelle entre Chifflet et le Dolois Louis Gollut ${ }^{30}$, dont Les Mémoires de la République Séquanoise, parues à Dole en 1592, avaient été interdites à Besançon. Pour les autorités bisontines l'ouvrage répandait,

$$
\begin{aligned}
& \text { plusieurs erreurs, mensonges, choses apocryphes, } \\
& \text { non véritables et aultres semblables contre les } \\
& \text { seurtez, droittures impériales, libertez, priviléges, } \\
& \text { antiquitez et franchises de cette cité }{ }^{31} \text {. }
\end{aligned}
$$

12 En effet, Gollut avait écrit que les habitants de Besançon soumis à la mainmorte, n'en n'avaient été délivrés que par l'intervention de Frédéric $\mathrm{I}^{\mathrm{er}}$. Besançon joue ici le rapprochement de Madrid tout en marquant l'éloignement de Dole. Tel est le sens du Grand Mémorial. Après la description juridique, est recréée une vaste fresque historique de la politique extérieure, accords et alliances, au cours des siècles, offrant dans un même élan des exemples multiples des vertus idéales de fidélité et amour que tout sujet doit offrir dans une relation contractuelle et personnelle avec son souverain. La démonstration de son courage militaire et de son poids décisif en repoussant l'envahisseur de toute la Bourgogne ${ }^{32}$, participent de l'entreprise de séduction orchestrée pour proclamer son affection à Madrid. Ici, le textesert l'ambition de son auteur : tout n'est que preuve et témoignage d'affection pour le roi d'Espagne, alors même que sont dépeintes les époques où la province a échappé à la souveraineté espagnole, tant et si bien que "no se puede distinguir del de los propios vasallos ${ }^{33}$ [que l'on ne peut le distinguer de celui de ses propres vassaux]. Le magistrat forme le dessein de supplanter le Comté, son concurrent dans le cœur royal, et entre en lice sur l'emblème de sa réputation : sa fidélité. L'amour du roi devient objet de compétition entre les deux entités politiques, ce qui est indice de son importance pour ces vassaux si éloignés. Le diplomate, Saavedra Fajardo proposait d'ailleurs le Comté comme modèle de fidélités ${ }^{34}$. En effet, le point culminant de l'expression de cette fidélité comtoise furent, sans conteste, les quatre-vingt jours du siège de Dole en $1636^{35}$. Siège au cours duquel les Dolois, les membres du Parlementdont le président, Jean Boyvin "âme de la résistance", ainsi qu'Antoine Brun, Procureur général et négociateur des traités de Westphalie ${ }^{36}$, et Ferdinand de Rye archevêque de Besançon, résistèrent avec bravoure et opiniâtreté aux assauts du prince de Condé et firent face aux ravages de la peste. Le retentissement de la victorieuse résistance doloise se fit connaitre à la cour de Madrid et dans toute la monarchie par la diffusion de plusieurs récits.

13 Les deux villes du Comté s'opposent dans une étroite compétition ayant pour objectif de recueillir les fruits de l'amour du roi, du père qui distribue charges et faveurs. Besançon s'arc-boute sur ses droits anciens pour raviver l'idéal de grandeur de la ville et de communion avec le monarque espagnol, gardien de la cité. La finalité de la stratégie bisontine est de s'inscrire dans une mémoire collective avec le souverain, ayant recours pour cela à cette histoire qui les soumettaient aux mêmes vicissitudes sous les règnes des monarques Habsbourg ${ }^{37}$.

Puis la cité proclame son autonomie en politique étrangère par son droit de conclure des accords diplomatiques à son entendement et libre arbitre ${ }^{38}$. C'est bien parce que la cité est suprême en sa juridiction, et que ce privilège lui fut constamment reconnu, qu'elle a pu 
conclure des alliances et traités avec le comte de Bourgogne, roi d'Espagne, depuis Charles Quint. La cité place ainsi la monarchie face à ses contradictions.Henry explicite cette stratégie rappelant au Roi qu'en signant le "Traité de Gardienneté», il reconnaissait de fait la capacité de la ville à signer des traités sans que l'Empereur n'ait à l'autoriser ${ }^{39}$.Cette recréation généalogique des batailles et traités est étayée par la multiplication documentaire. Les preuves sont, soit insérées dans le corps du texte du Grand Mémorial, soit adjointes en tant que compilationdes textes référencés en latin, langue d'empire et du droit. La qualité des preuves se fonde sur la forme des actes, ce qui permet de demander leur respect ${ }^{40}$. Les députés travaillent à diffuser ces textes et parviennent à intéresser les milieux de la cour à leurs revendications. Car après s'être hissée dans le concert des nations pour valider ses droits particuliers, Besançon par ces écrits reconstruit l'histoire du lien réciproque entrela cité et le comte de Bourgogne. C'est ainsi que la cité réactualise la solennité des actes et traités conclus et donne vigueur à leur mémoire commune. La légitimité qu'elle revendique est étroitement liée à une représentation idéale de son corps politique. Le Grand Mémorial, offre la vision des juristes de la cité sur leur existence historique face aux autres pouvoirs. Ils construisent leur propre système autoréférentiel s'appuyant sur la tradition et poursuivant un objectif précis : donner une valeur et une légitimité à des droits et à des privilèges qui pourraient être marchandés ultérieurement. Car ce dialogue, point de contact entre pouvoir central et autorités locales, va conduire la cité à penser une représentation de soi audacieuse en transcendant son propre cadre de références ${ }^{41}$. Luttant pour approcher le cœur du roi, écartant Dole de la proximité du souverain, le magistrat exporte son combat local au cœur de la monarchie. Il ouvre alors un chemin à ses prétentions qui font l'objet du point suivant. Comment Besançon prétend-elle résoudre cette entrée en monarchie, alors qu'elle doit coexister désormais, sur un plan local avec plusieurs autres pouvoirs rivaux, si ce n'est en réinterprétant l'héritage de la mémoire de la cité ?

\title{
Le transfert du parlement ou l'obtention de la capitalité par Besançon
}

Bruxelles avait informé les députés que le roi formait le projet de leur conserver,

\begin{abstract}
les droits et privilèges qui seroient compatibles avec la monarchie et quau regard de la surséance que nous demandions de l'execution de l'échange, il sen étonnait, les affaires se trouvant mieux accomodées en notre pays que par deça le parlement étant informé des droits de la cité ${ }^{42}$.
\end{abstract}

Naïveté ou mauvaise foi ? En effet, nul ne pouvait ignorer le degré d'hostilité entre les autorités des deux villes ${ }^{43}$. La controverse autour de la compatibilité des privilèges de la cité impériale au regard de la monarchie toute entière, et spécifiquement de Bruxelles et de Dole, demeure ouverte. Si pour la monarchie composée, réduire les enclaves territoriales signifiait introduire une simplification administrative, pour la cité impériale ce transfert de souveraineté induisait des menaces considérables en termes de distribution du pouvoir ${ }^{44}$. Car cela équivalait à placer les deux villes les plus importantes du territoire face à face, puis à poser la question de leur hiérarchisation juridictionnelle. Leur histoire commune, faite d'une interaction sous l'angle d'un rapport de force, nous fait entrevoir la portée de la mutation en cours. Se profilait donc l'intention de « réduire 
icelle cite a lentiere domination de VM comme lune des autres places du Comté de Bourgogne $»^{45}$. Ce transfert signifiait donc pour elle l'insertion dans un système polysynodal, sous l'autorité de Bruxelles, du gouverneur comtois et du Parlement. La représentation aux États de Franche Comté n'est pas mentionnée mais doit s'en inférer. La parole urbaine poursuit dès lors une finalité précise : éviter que le processus d'intégration ne soit un processus de fusion qui reviendrait à la placer, à l'introduire, dirions-nous, passivement dans un ensemble déjà construit et figé sur la plan juridictionnel. Concilier transfert et droits s'avérait ainsi un défi, un exercice de difficile équilibre, posé au pouvoir royal. Voici donc posée la problématique de cette intégration : Besançon est-elle soluble dans la province? La monarchie catholique peut elle lui garantir simultanément le respect de ses droits, tel qu'il était prévu dans l'acte de transfert de souveraineté de 1654 ?

17 Besançon ambitionne alors de modifier l'ordonnancement des pouvoirs dans la province. Par l'ouverture d'un espace transactionnel entre roi et cité, elle saisit l'opportunité de demander une réforme dans l'agencement des pouvoirs en obtenant un statut particulier au regard de leur architecture en Comté. À ce transfert de souveraineté sans contrepartie pour elle, Besançon répond par une initiative sur le mode de la transaction :

quayans de grans droits incompatibles avec la
souveraineté nous estions aussy prests de nous en
dépouiller au profit de Sa Majeste en contreschange
desquels nous supplions SM de nous vouloir accorder
le transport du parlement a la cité questoit plus a
l'advantage et utilite du service du Roy et de la
province qu'a celuy de Besancon ${ }^{46}$.

18 C'est là l'enjeu de leur stratégie au cours des années: l'obtention de la capitalité au détriment de Dole. Besançon reprend l'initiative amorcée sous Philippe II $^{47}$ et offre sa solution pour parvenir au dénouement de la crise ouverte: lier au transfert de souveraineté, le transfert du Parlement en ses murs. Par ce doublet audacieux, la primauté juridictionnelle de la cité serait alors garantie. Mais sa demande récurrente d'obtention du siège du Parlement équivalait à décapiter sa rivale Dole. Besançon n'obéira qu'à un Parlement siégeant en son sein, préviennent les députés, visée ambitieuse que résumait le Trésorier général à Bruxelles par ces mots de « novissisimi primi $»^{48}$.

19 À Bruxelles, Jules Chifflet ${ }^{49}$ avait secondé Henry et Belin en élaborant un plan de bataille cohérent. Des archives de Jean-Jacques, son père ${ }^{50}$ il avait exhumé une copie de la consulte rédigée en $1624^{51}$ par Pecquius et Boischot qui leur sera « utile parce qu'elle est absolument à l'avantage de la cité en ce qu'elle conclut au transport du Parlement dans la cité ». La preuve par l'incorporation d'anciens documents valide la résolution antérieure et traduit une constante mobilisation en faveur du dessein politique bisontin. Parallèlement, la continuité d'une lignée de serviteurs de l'état trouve là à s'affermir. La consulte de 1624 portait résolution sur des remontrances que Jean-Jacques Chifflet et Claude-Antoine Buson avaient présentées en ambassade à Bruxelles. Ils y défendaient, afin de prévenir toute incursion des hérétiques français, une union entre ceux de Besançon et ceux de Bourgogne par le transfert du Parlement en vertu d'un « emprunt de territoire » qui garantissait le statut juridique de la cité. En effet le Parlement avait été ambulatoire $^{52}$ sous les ducs et comtes de Bourgogne, puis établi à Dole. L'archiduc Albert avait repris ce même dessein en envoyant dans la cité impériale des commissaires pendant une année entière et qui, à leur retour, lui avaient présenté un rapport favorable à son projet ${ }^{53}$. L'avantage était le suivant : si le comte de Bourgogne et le protecteur de la 
ville devenait une seule et même personne, on ne pourrait craindre de la voir préférer un roi étranger comme gardien, comme le fut Louis XI pendant treize ans ${ }^{54}$. Ceux de Dole surent défendre l'idée de la complexité de la réforme puisque l'emprunt de territoire signifiait que ce Parlement doté de tant de lustre siégerait en ville étrangère !

\section{Le serment comme menace de fronde}

Intégrer la ville la plus riche et la plus prospère comme un simple agrégat au sein du Comté de Bourgogne, sous-entendait donc sa soumission au pouvoir judiciaire du Parlement de Dole, au gouverneur général des Pays-Bas et de Bourgogne, anéantissant par là même son antique autonomie juridictionnelle. En contrepartie, cela signifiait un accroissement des pouvoirs juridictionnels du Parlement et des Pays-Bas. Aussi le magistrat réclame son droit à être entendu avant que la cession ne soit conclue à son désavantage. En réclamant ce qu'il nomme " une surscéance de l'exécution », il tente de bloquer la décision d'envoyer le marquis de Saint-Martin, gouverneur de Dole, à Besançon pour y recevoir le serment de fidélité et d'obéissance, et ce jusqu'à ce que Philippe IV prenne connaissance de ses suppliques. Jules Chifflet occupe une première place dans la mise en œuvre de cette stratégie de rapport de force et de confrontation ${ }^{55}$. Ainsi le rapportent les deux députés :

il nous dit de parler hardiment que cestoit le coup de la partie parce quayant presté serment nous ne serions pas dans la liberté de parler comme nous sommes a présent quil ne fallait rien apprehender de pousser laffaire iusque au bout ${ }^{56}$.

C'était là une dangereuse incitation à la désobéissance. Cette menace de non exécution de l'ordre de prêter serment replace Bruxelles dans la hiérarchie des pouvoirs; les députés rappellent le pouvoir supérieur qu'est Madrid, auquel Bruxelles est soumis, et en appellent à la justice du roi qui ne peut qu'entendre leurs légitimes remontrances ${ }^{57}$. Il s'agit alors de prendre de vitesse l'envoi de la commission à Dole pour prévenir la rébellion en accourant à Madrid. Yvan Lignereux le montre clairement, il s'agit

$$
\begin{aligned}
& \text { de passer par-dessus la perversion du médiateur } \\
& \text { altérant la représentation même, pour s'adresser au } \\
& \text { représenté, en partant du principe que celui-ci était } \\
& \text { également trahi par son représentant }{ }^{58} \text {. }
\end{aligned}
$$

Le magistrat veut court-circuiter ce pouvoir intermédiaire qu'est Bruxelles et son relais direct en Comté qu'est le gouverneur. Alors que pour le marquis de Caracena , gouverneur des Pays-Bas et de Bourgogne, il fallait exécuter sans délais l'ordre royal donné le 7 juin 1660, comme l'en enjoignent avec insistance les missives royales. Cet ordre consiste à prendre possession de la cité, à obtenir qu'elle prête serment de fidélité au roi, ce qui marque l'étape de son intégration dans le corps de la Bourgogne et rend effective l'obligation réciproque entre cité et souverain ${ }^{59}$. Mais la hiérarchie de délégation se révèle illégitime aux yeux des citoyens de Besançon ${ }^{60}$. En effet, il demeure inconcevable et même injurieux pour les citoyens de la cité de prêter serment à des sujets qui sont leurs égaux. La préséance entre villes du Comté de Bourgogne, notamment lors de la tenue des États, ouvrait déjà le champ à une kyrielle de disputes. Aussi, prétendre imposer une nouvelle géométrie des relations entre citoyens bisontins et sujets comtois pour des hommes qui n'avaient eu que des rapports d'égalité à travers des relations de ville à province était risqué. Les gouverneurs se trouveraient placés de fait sous l'autorité du Parlement, ce qui leur était insupportable. Le refus d'obtempérer, tenait également au 
contenu de la lettre de commission qui recélait une faille. Bruxelles, dans le pouvoir établi pour le gouverneur de Dole, lui conférait l'administration de la police et justice, au-delà donc de la simple prise de possession de la cité. La vive dénonciation de Besançon illustre le soin jaloux avec lequel le magistrat veillait à la stricte délimitation de la compétence de chaque juridiction et son combat permanent contre tout empiétement ou accroissement des juridictions adverses. Le marquis de Caracena, lui, brandit cet ordre comme menace pour obtenir la reddition juridique de la cité . Il assure que prêter serment lui ouvriraitles portes de la grâce et faveur royale,

$$
\begin{aligned}
& \text { estant notoire que la revocation dicelle conferera } \\
& \text { mauvais exemple et que la desobeissance de } \\
& \text { quelquuns peu affectionnés obtiendront la fin de } \\
& \text { leurs intentions reduisant la grandheur de VM a des } \\
& \text { conditions }^{61} \text {. }
\end{aligned}
$$

Le 5 janvier 1661, le marquis de Caracena transmit un pouvoir au marquis de Saint Martin pour recueillir le serment de la ville. Il en informait les gouverneurs de Besançon et demandait au Parlement d'assister de ses conseils le marquis de Saint Martin pour la prise de possession. En liant ainsi le corps du Parlement à la cérémonie du serment, il démontre que le Parlement ne pouvait être ignoré de cette agrégation. Mais le magistrat éconduit le marquis de Saint Martin qui essuya alors un affront public. Le Parlement informa Bruxelles des grandes oppositions rencontrées par Saint Martin, concrétisées par ce refus d'obtempérer, qui l'avait obligé à se retirer après avoir lu et déposé en place publique, devant l'Hôtel consistorial, les articles de la prise de possession. Le Parlement peut alors écrire à Caracena tentant de reprendre l'avantage :

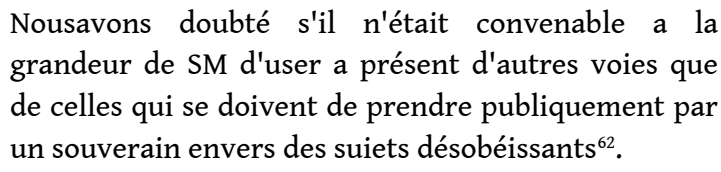

Le Parlement redouble d'agressivité en proposant que les Bisontins soient déclarés sujets rebelles et désobéissants et que leurs biens soient confisqués en Comté. LeParlement s'engouffre ainsidans la brèche qui semble lui offrir la possibilité d'en finir avec son rival politique.

La question du serment, engagement verbal actant le consensus politique et marqueur de continuité de la relation contractuelle souverain/sujet, trouvait lors de cette ambassade toute son importance, puisqu'elle comportait un risque. En effet, il pouvait devenir l'étincelle qui allumerait la flamme de la désobéissance, par laquelle les gouverneurs s'affranchiraient de leurs protestations convenues de réjouissances de cette nouvelle souveraineté $^{63}$. À partir de cet affront aux pouvoirs concurrents, se déclenche un processus dont les conséquences pourraient mettre en péril un équilibre des pouvoirs fragilisé par l'éloignement structurel du centre et le manque de ressources actuel de ces territoires du nord. En rejetant le serment de fidélité au Roi, le magistrat prouve sa capacité à désobéir et à rejeter l'autorité conjointe de Bruxelles et du Parlement. Il ordonne à ses députés en cour de,

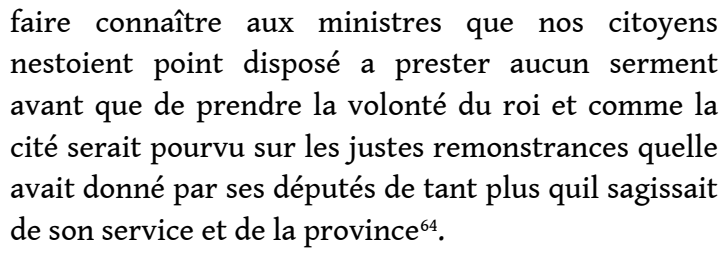


lors, Besançon doit affronter l'animosité qui se répand en cour et reprendre l'initiative pour ne pas perdre sa légitimité. En effet, la nouvelle du refus d'obéissance de la cité aux sommations du marquis de Saint Martin, «fut trouvé extraordinaire dont pourtant il ne falloit faire grand bruit» ${ }^{65}$, contraint le magistrat à adopter une attitude de retrait pour atténuer son retentissement néfaste sur l'image de la cité. Il propose alors de faire tenir une prestation de serment immédiate entre les mains royales. Tout en manifestant dans ses lettres sa clairvoyance sur les inconvénients qu'il y avait à prêter serment avant d'être entendu ${ }^{66}$. Il s'agit donc bien d'un recul prudent. Le magistrat fait parvenir à ses députés un factum justificatif relatant les circonstances de ce qui est désigné en cour sous le nom de fronde, ainsi qu'un pouvoir déléguant faculté de prêter sur le champ le serment de fidélité refusé localement. Le 27 mai 1661, ils remettent au Roi un mémoire pour être admis au serment de fidélité.

\section{Conclusion}

On ne sait à qui vous êtes, estants démembrés de l'empire et nayant pas prestés serment, vous n'estes ni à Dieu ni au Diable ${ }^{67}$.

C'est alors qu'éclate la colère des ministres. Devant le blocage créé par la cité impériale qui résiste à devenir royale, ils ne consentirent pas à « admettre la cité au serment soub la réforme de ses droits car ils desiroient que le Roy soit un roi de pointure ${ }^{68}$. En conclusion, nous pouvons dresser un bilan sur la capacité d'inflexion de la ville sur cette décision politique d'agrégation la concernant.

En premier lieu, Besançon est parvenue par son ambassade à créer un lien d'immédiateté avec le Roi quand bien même le serment n'avait pas encore été prêté. Philippe IV prend ainsi place dans la continuité de la souveraineté de l'empereur. Écartant les autres acteurs des composantes territoriales de la monarchie, elle s'est frayé un passage entre ministres et conseillers, recueillant alors une parole royale de reconnaissance personnelle "Tendré cuidado ${ }^{69}$. Le lien politique fondé sur la réciprocité, ainsi actualisé, fait entendre que Philippe IV entendra le député de cette lointaine cité qui se présente à lui pour obtenir un arbitrage en sa faveur. Mais ces mots d'autorité plaçaient le requérant en position d'attente, détruisait la proximité royale et renvoyait la cité à l'autorité des conseils et ministres de la polysynodie. C'est ainsi que le Grand Mémorial est remis au Conseil des Flandres et qu'est formée une junte qui ouvre la voie au traité de 1664.

Car si la cité a démontré que le roi ne pouvait prétendre d'autres droits que ceux que lui avait cédés l'empereur, parallèlement, elle a revendiqué l'accrois-sement des siens en conditionnant son intégration pleine et entière dans le Comté de Bourgogne. Afin que cette incorporation s'effectue également à son profit, Besançon a louvoyé entre diplomatie au-delà et fronde en deçà, tentant d'évincer les conseils de la polysynodie par son droit d'immédiateté au prince. Là est mis à jour son second gain politique, Besançon est parvenue à créer un espace politique pour que l'appareil administratif entende ses droits et prétentions en reprenant son ancienne demande de transfert du Parlement.

Le traité de 1664 conclu par l'entremise du marquis de Castel Rodrigo compensera la perte des droits de la cité par un accroissement de sa juridiction au détriment du Comté. Si intégrer les nouveaux territoires en maintenant leur modèle politique favorisait la loyauté future des élites, dans son étroite marge de manœuvre entre querelles juridictionnelles ${ }^{70}$, le roi opte pour un compromis tranchant ainsi :

Les Cahiers du Centre de Recherches Historiques, 44 | 2011 
Yo no quiero fazer tanto agravio a mis fieles ny por Bisancon ny por todo el mundo queda el Parlamento en Dola y a Bisancon se de todo el que se puede ${ }^{71}$.

\section{NOTES}

1. Les Comtois signent l'acte de capitulation en février 1668, prêtent serment et passent sous souveraineté française pour quatre mois jusqu'au Traité d'Aix-la-Chapelle, par lequel, monnaie d'échange diplomatique entre les deux Couronnes, la Franche-Comté est restituée à l'Espagne. En 1674 nouvelle campagne militaire française et passage sous domination modèle louis quatorzien par le traité de Nimègue.

2. Conquête malgré la quasi-absence de résistance en 1668 , nous nuancerons notre propos pour 1674.

3. Les enquêtes lancées par des commissaires flamands en 1668 sont la preuve de la suspicion générale.

4. Signé entre le marquis de Castel-Rodrigo à Besançon en chemin vers Bruxelles où il prenait la charge de Gouverneur des Pays-Bas et de Bourgogne.

5. À Besançon, alors que le Parlement siégeait à Dole (après avoir été ambulatoire) et que le Gouverneur, un Bauffremont résidait en son Hôtel de Gray.

6. Pendant la Guerre de Trente Ans, les Espagnols avaient conquis la ville forteresse de Frankenthal dans le Palatinat sur les Suédois et lors du Traité de Munster, Philippe IV et Ferdinand II en décidèrent l'échange. Le 4 décembre 2009, Luis Tercero Casado (Universität Wien) a présenté La Paz de Westfalia inconclusa: España y la restitución de Frankenthal (1649-1653) au Congreso Internacional : La Dinastía De Los Austrias : la Monarquía Católica y el imperio (siglo XVII) tenu à Madrid.

7. Mais connu par la ville grâce à la correspondance avec François de Lisola de Vienne, 1645/1653. Archives municipales de Besançon, AA 32. folio 2-9. [Désormais le sigle AA 32 désigne les archives municipales de Besançon]

8. Image récurrente du comté de Bourgogne. Cf. Girardot de Nozeroy. Histoire de Dix Ans de la Franche-Comté de Bourgogne : (1632-1642), Besançon, Manuscrit publié par Jules Crestin, 1843.

9. Le 4 mai 1534 l'empereur Charles Quint, roi d'Espagne et comte de Bourgogne renouvela le traité de Gardienneté avec la cité. Émerge ici un ressort complexe de la situation de la province, ni territoire stable, ni territoire homogène: terre enclavée et terre d'enclaves, dont Besançon la ville la plus peuplée, 13000 habitants contre 5000 habitants pour Dole au XVI ${ }^{e}$ siècle. En outre Besançon et le Comté font partie du Cercle de Bourgogne créé en 1548, grande unité régionale des Pays-Bas et du Comté avec le Saint Empire.

10. Alicia Esteban Estringana,Madrid y Bruselas. Relaciones de gobierno en la etapa postarchiducal (1621-1634),Louvain,Presses Universitaires de Louvain,2005. Définissant le partage juridictionnel entre province et souverain, il est reconnu que le gouverneur du Comté, «a mantener comunicación directa con Madrid presentaba una incuestionable subordinación a Bruselas ", p. 38 [qui malgré le maintien d'une communication directe avec Madrid, présentait une subordination incontestable à Bruxelles]. Le roi se réserve la nomination du président du Parlement, du gouverneur ainsi que de l'archevêque de Besançon. Au gré des relations personnelles avec le gouverneur des Pays-Bas (le marquis de Caracena (1659-1664), puis le marquis de Castel Rodrigo (1664-1668), les ordres de Bruxelles sont exécutés ou non. 
11. Lettre de Madrid du 27 juin 1660, Besançon, Bibliothèque d'étude et de conservation de Besançon, AA10, folio 2. L'empereur Léopold pour sa part avait libéré la cité de l'obéissance et la sujétion à laquelle ils étaient tenus, leur demandant de reconnaître comme prince Philippe IV et de s'y porter d'autant plus volontiers que précisément leurs droits et privilèges avaient été conservés par le Traité d'échange. Lettre de Vienne en latin du 4 novembre 1660. Ibid., folio 3.

12. John H. Elliott. «A Europe of composite monarchies », Past \&Present, 137, 1992, p. 48-71.

13. Tandis que Bruxelles tentera de décrédibiliser le magistrat par cette question de la représentation.

14. Ainsi est mise en lumière toute une sphère de pouvoirs reliés sous la titulature de « Gouverneur des Pays-Bas et de Bourgogne ».

15. Comme le portent les "Délibérations municipales", dont les registres sont conservés à Besançon. Chaque bannière élisait quatre citoyens sindics, ceux-ci choisissaient leur Président et élisaient les quatorze gouverneurs, souverains en matière d'administration et police. Ils jugeaient avec l'assistance du juge que le souverain du comté de Bourgogne entretenait dans la cité. En matière civile, seul le conseil aulique de l'Empereur pouvait révoquer leurs sentences. En 1660 pour cette question, il fut décidé de porter le nombre à 42. Par un arrêt royal de 1676, l'ancienne forme du magistrat de Besançon fut abolie. Notes sur l'Histoire municipale de Besançon, Besançon, Castan, 1898, p. 419-420.

16. Commentaires Jules César, I, 8 XXXVIII, « [...] ad occupadumVesuntionem, quod est Oppidum maximum Sequanorum contendere ».

17. Grand Mémorial : 33 pages imprimées en espagnol, bibliothèque d'étude et de conservation de Besançon, collection Chifflet 72, 110.

18. «juridiccion de su magistrado que ella elige a su arbitro y nombra anualmente, no puede sin infraccion de sus derechos, estar subordinada a nigun otro tribunal o sea consejo, que solo a la Real persona de Su Majestad, segun lo era del Señor Emperador antes de la cession y segun viene dispuesto en ella. Tiene dicha Ciudad esta juridicccion suprema desde el principio de su fundacion ", p. 1. [la juridiction de son magistrat qu'elle se choisit selon son libre arbitre et nomme anuellement, ne peut sans infraction de ses droits, être subordonnée à aucun tribunal ou conseil, mais seulement à la royale personne de SM, tel qu'elle l'était de l'Empereur avant la cession et tel que prévu à cet effet] ibid.,Grand Memorial, copies françaises imprimées, folio 257 507, p. 1.

19. «[...] jamas ha reconocido ninguna Camara Imperial de Alemania, ni la de Spir (a la qual quedan subordinadas los Principes Electores, y demas Ciudades del Imperio) »,Ibid., p. 5 [elle n'a jamais reconnu aucune Chambre impériale d'Allemagne, ni celle de Spire (à laquelle demeurent subordonnées les Princes électeurs et les autres villes de l'Empire], p. 1.

20. «Señor, La Ciudad de Bisanzon dice que [...] ». (Ainsi traduit dans les copies en français), ibid. , Grand Memorial,folio 257.

21. «con aprobacion de los Señores Emperadores, y V. Mag. Mismo, y todos sus gloriosos Protegidores Reyes de España, y Duques de Borgoña, reconociendola por Ciudad de juridicion Suprema, [ ...]», [avec l'approbation de l'Empereur et de Sa Majesté et de tous ses glorieux protecteurs rois d'Espagne et ducs de Bourgogne, la reconnaissant comme cité de juridiction suprême], Grand Memorial, op. cit.

22. "Qu'il s'agisse du fantôme de la Rome impériale ou de la Rome bien vivante des souverains pontifes", in Alain Tallon, Le sentiment national dans l'Europe méridionale aux XVI et XVII siècle, Madrid, collection Casa Velazquez, 2007, Introduction p. XV.

23. La restitutio peut s'analyser au final comme une opération de captatio et de traslatio de l'autorité du passé sur le présent, mais au nom d'un passé que l'on fait sien ", François Hartog, «L'autorité du temps ", Études. Juillet-août 2009, p. 51-64, (en part.)p. 54.

24. «Tiene dicha Ciudad esta juridiccion suprema desde el principio de su fundacion » [Cette ville possède cette juridiction suprême depuis sa fondation] . « ha conservado esta suprema juridicion, 
y derecho de hermandad con el favor singular de Dios(aviendo sido une de la primeras Ciudades del mundo que abraço la Santa Fe Catolica y inquisicion) ha gozado la dicha de no aver sido jamas sujetada de ninguna provincia (ventaja sobre la misma Roma ) antes resistiendo a todas las invasiones de sus enmigos " [elle a conservé cette juridiction suprême .par la faveur singulière de Dieu ( ayant été l'une des premières villes du monde qui embrassa la sainte Foi catholique) elle a bénéficié du bonheur de n'avoir jamais été soumise à aucune province ( avantage qu'elle possède sur Rome même) ayant résisté à toutes les invasions de ses ennemis.]. «Bisanzon fue siempre la Metro-poli de la Sequania hasta que adquirio el derecho municipal de Roma»[Besançon a toujours été la métropole de la Séquanie jusqu'à ce qu'elle acquière le droit municipal de Rome]», ibid., Grand Mémorial, p. 2- 4.

25. De droit des citoyens à exercer charges et dignités dans le Sénat romain sans en retour imposition au droit d'élection de son magistrat accordé à tous les citoyens de la cité, en faisait une démocratie.

26. Hans Bots, Françoise Waquet, La République des Lettres, Paris, Belin, 1997.

27. Lors de son ambassade auprès de Philippe IV en 1626 pour obtenir le transfert du Parlement à Besançon. Ibid, Collection Chifflet 25, folio 187, 269. 24 folio 26.Les liens personnels établis auprès du Roi sont très souvent occasion d'octroi de charges titres et faveurs garant de l'amour réciproque futur. Pour les nobles bourguignons est très prisée la Toison d'Or.

28. La province est appelée Séquanie, de la source du même nom dans la montagne du Jura signifiant temple ou sacré, car la religion comme l'a fait remarquer César y fut toujours observée. «de tan larga Provincia ninguno niega que Bisanzon fundo los principios y tuvo la superioridad " et "Vesontionem Sequanorum urbem primum aedificari caeptam asserunt", [d'une si longue province, personne ne nie que Besançon en fonda les principes et en eut la supériorité], ibid.,Grand Memorial, p. 3.

29. Les nombreux essais sur les antiquités de Besançon montre le choix du passé dans lequel la communauté des savants et érudits veut se reconnaître et le proposer à la société comme modèle. 30. Louis Gollut, (1535-1595), inventorie des documents pour mener à bien son œuvre maîtresse mise sous presse à Dole en 1591, Les mémoires historiques de la République séquanoise et des Princes de la Franche-Comté de Bourgogne. Dans l'émeute nocturne du 10 juin 1668 fut pillée la maison de son fils conseiller au Parlement et sa bibliothèque jetée à la rue, fut dispersée.

31. Ibid.,Louis Gollut, p. XI

32. Vertus auxquelles elle ajoute sa supériorité militaire pour marquer un point en terme stratégique de défense des territoires du nord.

33. "Y ultimamente en las guerras passadas, en las cuales ha dado a V. Mag. tales pruebas de su zelo, y amor, que no se puede distinguir del de los propios vasallos » [et dernièrement dans les guerres passées durant lesquelles elle a donné à SM de telles preuves de zèle et d'amour que l'on en peut le distinguer de celui de ses propres vassaux], Grand Memorial, op.cit., p. 2.

34. Saavedra Fajardo, "Ideas de un principe cristiano representado en cien empresas» et s'inquiétait de l'incorporation de Besançon dans sa Relacion envoyée à Philippe IV en 1638.

35. «Sus Majestades salieron el domingo a dar gracias a nuestro Señor por las buenas nuevas en pùblico. Se dice que es por lo del condado de Borgona, y por estar Dola descercada, con grande perdida de los franceses ». Le Roi apercevant deux religieux bourguignons s'écria : « Por Borgona, por Borgona»,Lettre de Madrid, 23 septembre 1636 du père Sebastian Gonzalez S.J. au père Rafael Pereyra S.J.( CCCXCVVII), Mémoires et documents inédits pour servir à l'histoire de la Franche-Comté, t. X, Besançon, 1912, p. 563.

36. Antoine Brun s'opposa à la restitution de Frankhental au nom du roi d'Espagne et introduisit le thème de l'échange. Truchis de Varenne, Antoine Brun, Besançon, 1932.

37. Comme un processus d'acquisition d'une tradition composée de couches successives et modelant les contours d'un savoir consensuel que le Roi ne peut rejeter. Le rappel de la Guerre de Dix ans qui a vu la population du Comté disparaître des deux tiers concourt à cet objectif. 
38. "Conservado con su propio valor y usado continuamente su juridiccion suprema con aprobacion de los Senores Emperadores y VM mismo han tratado con ella sin participacion del Imperio, y concluido las alianças, y confederaciones, que son tan notorias ». [Conservant par son propre courage et usant sans discontinuité sa juridiction suprême avec l'approbation des empereurs et de SM ont signé des traités avec elle sans la participation de l'Empire, et conclu des alliances et confédérations qui sont notoirement connues], ibid., Grand Memorial.

39. «Et mesme SM par le Traité de gardienneté fait avec la Cité la reconnu pour sa supreme juridiction puisquelle la passe sans participation de l'Empereur ou de l'Empire ny ayant heu aucune puissance ou souverain quy ayt ecercer la supreme juridiction dans la Cité non pas mesme les Empereurs Romains ", « Négociations faictes en cour de Bruxelles/sur le voyage/ $3^{e}$ cahier : "Négociations faictes en cour de Madrid en l'an 1661", Besançon, bibliothèque d'étude et de conservation de Besançon, AA. 33. folio 1-4.

40. Martine Grinberg, Écrire les coutumes. Les droits seigneuriaux en France, Paris, PUF, 2006. Puiser dans les archives la trame continue d'une histoire sans faille rend compte de la capacité des magistrats lettrés à orienter en leur faveur, par des arguments réfléchis, l'expression politique du corps de la ville.

41. Rosario Villari, « La idealizacion del pasado y el tradicionalismo no impidieron la bùsqueda y la afirmacion de un nuevo independenismo, distinto e incluso opuesto al independentisme tradicional », Revoluciones periféricas. 1640 : La Monarquía hispánica en crisis,1991, p. 175.

42. Ibid., AA 46.

43. Comme lors de la récente affaire de l'annotation des biens des gouverneurs en Comté par le Parlement, en rétorsion aux scellés posés par le magistrat sur la maison de feu le gouverneur et rendant donc inaccessible ses papiers d'état. Archives du Parlement de Dole, 2B 392.

44. José Javier Ruiz Ibáñez, Felipe II y Cambrai : el consenso del pueblo,Madrid, Sociedad Estatal para la Conmemoracion de los Centenarios de Felipe II y Carlos V, 1999.

45. Ibid., AA33, Advis de Conseil d'Etat de Flandresdaté du 4 mai 1661.

46. Ibid., Petit cahier de Belin, AA33, folio 3.

47. Christian Windler. "Städte am Hof. Burgundische Deputierte und Agenten in Madrid und Versailles (16.-18. Jahrhundert)», Zeitschrift für Historische Forschung,30, 2003, p. 207-250.

48. Sic erunt novissimi primi et primi novissimi , Mathieu XX 16.

49. Docteur en droit nommé Chancelier de l'ordre de la Toison d'or par Philippe IV et conseiller au Parlement de Dole en 1659. Écrivit ses Mémoires qui couvrent notre période : 1668-1674 et qui furent publiées dans laCollection Mémoires et documents inédits pour servir à l'histoire de la FrancheComté ; V et VI, Besançon, Impr. Outhenin-Chalandre fils, 1867-1868 .

50. La volumineuse et éclectique Collection Chifflet alors en cours de constitution, maintenant conservée à la Bibliothèque de Besançon et numérisée en 2009.

51. Consulte du 12 mars 1624 adressée à l'Archiduchesse Isabelle, Besançon, Collection Chifflet, 39.

52. «L'ordonnance de l'Empereur Charles V faiste l'an 1539 ou il est dit par expres que le Parlement de Bourgougne sera seant en la ville de Dole, si pour bonnes considerations ou par ses successeurs il nest autrement ordonné, laquelle reserve demonstre evidement l'intention secrette des Princes de la placer a Besançon quand l'occasion s'en presenteroit » On aurait, quant à nous, préféré souligner le dernier segment de phrase ! Ibid., Collection Chifflet 26, folio 487.

53. Ibid., "qu'il étoient de tout expédient de faire ledit transport lequel conferoit bientôst une telle union desdits de Besançon et de ceux dudit Pays, que ce ne seroit plus qu'une mesme chose "

54. Ibid., «Les successeurs comtes de Bourgogne seront dorénavant protecteurs héréditaires de laditte cité [...] il ny aura plus de crainte que ceux de Besançon a l'advenir preignent d'autres protecteurs estrangers; coils ont fait autrefois le roi de France Louis X l'espace de treize ans, des comtes de nevers et autres, ce qui serait a present beaucoup plus dangereux quil n'estoit 
autrefois, [...] avec telz avantages es autres articles, il ne restera a V.M. Que le tiltre de souverain, puisque par effet, il aura tout le reste ".

55. Cela nous permet d'établir à sa juste mesure le prestige dont jouissait Jules Chifflet, dépositaire et maillon intellectuel de la dynastie Chifflet auprès des Bisontins avant même qu'il n'occupe de retour dans sa petite patrie un rôle lors des événements de 1668.

56. Ibid., AA 33, cahier 1.

57. Continuité diplomatique s'inscrivant dans les pas de l'ambassade de Chifflet et Buson d'autant plus que la consulte de 1624 des conseillers des Pays-Bas les assurait de leurs droits.

58. Yvan Lignereux, Lyon et le roi : de la bonne ville à l'absolutisme municipal, 1594-1654, Paris,Champ Vallon, 2003, p. 425.

59. Barbara Stolberg-Rilinger, «Le Rituel de l'investiture dans le Saint-Empire », Revue d'Histoire moderne et contemporaine, 2009-2, p. 7-28.

60. Le tracé formel d'autorité était validé par les procurations: Saint Martin recevant commission du Roi par subrogation de Caracena.

61. AA33 folleto 47 en espagnol, « siendo notorio el mal exemplo que causara la revocacion della y que la inovediencia de algunos poco afectos logra el principal de sus intentos reduciendo a condiciones la grandeza de VM» el marques Conde de Pinto. consulta del marqués de Caracena. 4 mai 1661 et folleto 46 : [Estant notoire que la revocation dicelle confera mauvais exemple et que la desobeissance de quelquns plus affectionnes obtiendront la fin de leurs intentions reduisent la grandheur de V M a des conditions].

62. 14 avril 1661, Besançon, archives du Parlement de Fole, 2B 381-283.

63. Ana Isabel Carrasco Manchado:«Palabras y gestos de compromiso : los reyes castellanos y sus juramentos (siglo XV) »,e-Spania, 4, décembre 2007.

64. Archives municipales de Besançon, Minutes des résolutions prises par les vingt huit députés de l'assemblée générale de la cité impériale, 1661-1662.

65. Ibid., AA 46.

66. Ibid., Lettre du 15 mai 1661.

67. AA 46.

68. Ibid., et archives du Parlement de Dole, 2 B. 382.

69. « qui est a dire j'en aurai du soing » ainsi traduit par le député dans son petit cahier.

70. fiscales notamment : la hausse de l'impôt sur le sel.

71. [quest a dire e ne veu point causer un si grand prejudice a mes fidéles vassaux que le parlement demeure a Dole et a Besancon quon luy donne tout ce qui se peut].

\section{AUTEUR}

ANNONCIADE DE CAMBOLAS

EHESS/CRH 